\title{
VULNERABILIDAD PARA SIDA EN MUJERES QUE EJERCEN LA PROSTITUCIÓN EN SEVILLA
}

\author{
Taciana Silveira Passos ${ }^{1}$ \\ Nuria Cordero Ramos ${ }^{2}$ \\ Cristiane Costa da Cunha Oliveira ${ }^{3}$
}

Resumen: El objetivo de este trabajo fue relatar factores asociados a vulnerabilidad para VIH/SIDA en mujeres en dificultad social que ejercen la prostitución en Sevilla-España. Se realizó un estudio cualitativo, consistentes en revisión de fuentes bibliográficas y relato de observaciones en un proyecto de Atención Social a Mujeres prostituidas de una Organización No Gubernamental (ONG) de España con oficina en Sevilla. Se procedió a realizar el trabajo de campo entre el abril y mayo de 2017 y se utilizó un cuaderno de campo para el registro de la información, la muestra fue compuesta por 15 participantes que cumplieron criterios de elegibilidad. Las mujeres en situación de prostitución atendidas por la ONG proceden de entornos de pobreza estructural, lugares donde faltan los recursos básicos, de lugares en conflicto o donde han sufrido violencia. No obstante, la prostitución profundiza aún más esta situación. La condición femenina marcada por la mala situación económica se consideró, en este estudio, como un determinante de la vulnerabilidad social evidenciado por factores asociados, como el uso de drogas, sometimiento a la pareja sexual, la dependencia financiera y económica y la cuestión de la supervivencia. La vulnerabilidad programática fue identificada en la falta de conocimiento de los programas de prevención de Infección de Transmisión Sexual, en los aspectos de la maternidad y la salud reproductiva y el impacto del diagnóstico del VIH. A partir de los resultados de ese estudio, se puede

\footnotetext{
${ }^{1}$ Filiada ao Programa de Pós-Graduação em Saúde e Ambiente da Universidade Tiradentes

${ }^{2}$ Doctora en Derechos Humanos y Desarrollo por la Universidad Pablo de Olavide; Profesora Titular del Departamento de Trabajo Social y Servicios Sociales de la Universidad Pablo de Olavide (Sevilla)

${ }^{3}$ Doctora en Salud Colectiva, Docente de Programa de Posgrado en 'Saúde e Ambiente' de la Universidade Tiradentes
} 
observar la necesidad de crear programas de prevención y promoción teniendo en cuenta la vulnerabilidad social, que incluyan intervención en solución de problemas y estrategias de afrontamiento.

Palabras-Clave: Síndrome de Inmunodeficiencia Adquirida. Vulnerabilidad Social. Grupos de Riesgo. Trabajo Sexual.

Abstract: The objective of this study was to report factors associated with vulnerability to HIV/AIDS in women in social difficulty who practice prostitution in Seville-Spain. A qualitative study was carried out, consisting of a review of bibliographical sources and an account of observations in a project of Social Assistance to Prostituted Women of a Non Governmental Organization (NGO) in Spain with an office in Seville. Field work was carried out between April and May 2017 and a field notebook was used to record information. The sample consisted of 15 participants who met eligibility criteria. Women in prostitution served by the NGO come from environments of structural poverty, places where basic resources are lacking, places in conflict or where they have suffered violence. However, prostitution further deepens this situation. In this study, the female condition marked by a poor economic situation was considered a determinant of social vulnerability evidenced by associated factors, such as drug use, sexual partnering, financial and economic dependence and the question of survival. Programmatic vulnerability was identified in the lack of awareness of Sexually Transmitted Infections prevention programs, aspects of motherhood and reproductive health, and the impact of HIV diagnosis. Based on the results of this study, it is possible to observe the need to create prevention and promotion programs taking into account social vulnerability, including intervention in problem solving and coping strategies.

Key Words: Acquired Immunodeficiency Syndrome. Social Vulnerability. Risk Groups. Sex Work.

\section{Introducción}

La infección con el virus de la inmunodeficiencia humana (VIH) ha alcanzado en todo el mundo, un número alarmante. Las últimas estadísticas del 
Programa del VIH de las Naciones Unidas (UNAIDS) informan que aproximadamente 7.000 personas están infectadas con el VIH, a diario, y una persona muere cada 20 segundos de una enfermedad relacionada con el Síndrome de Inmunodeficiencia Adquirida (SIDA) y que es considerada la quinta causa de muerte entre los adultos (World Health Organization, 2016). La carga sanitaria y la carga socioeconómica generada por estas infecciones afectan, no sólo a los países en desarrollo, sino también a los países con mayor nivel económico.

En España, los datos del Ministerio de Sanidad, en 2014, muestran que fueron 3.366 casos positivos de VIH diagnosticados, casi diez cada día. La tendencia sigue a la baja entre los nacidos fuera de España, aunque suponen casi uno de cada tres nuevos afectados. No obstante, esta media es superior entre las mujeres, donde las inmigrantes son más de la mitad, un hecho que puede explicarse por la condición de víctimas de explotación sexual de muchas de ellas. Tras los españoles, la mayor tasa se registra entre la población latinoamericana $(15,1 \%)$ (Ministerio de Sanidad, 2016).
El concepto de vulnerabilidad en la salud pública fue primero asociado, por Mann, Tarantola y Netter, a partir de 1993, en el contexto de epidemia del SIDA. La adopción del término vulnerabilidad permite traducir la complejidad de los aspectos individuales y colectivos relacionados con la exposición al VIH y la enfermedad por SIDA (Mann et al., 1993). Sustituye las nociones de grupos de riesgo y las de comportamiento de riesgo. Asociadas a ideas rotuladoras $\mathrm{y}$, por lo tanto, generadoras y reproductores de prejuicio y estigmatización. En este sentido, se destaca el esfuerzo teórico de un grupo de investigadores en la elaboración de un marco de referencia conceptual y metodológico capaz de captar y evaluar vulnerabilidades en niveles interdependientes de análisis: individual, social y político-programático (Ayres et al., 2016a).

La vulnerabilidad individual comprende los aspectos biológicos, emocionales, cognitivos, actitudes $\mathrm{y}$ referentes a las relaciones sociales. La social se caracteriza por aspectos culturales, sociales y económicos que determinan las oportunidades de acceso a bienes y servicios. La vulnerabilidad programática se refiere a los recursos 
sociales necesarios para la protección del individuo a riesgos a la integridad y al bienestar físico, psicológico y social. Según los mismos autores, la vulnerabilidad depende de la combinación de los elementos de los tres dominios en el momento actual. Depende también de las experiencias relativas a cada uno de ellos en el pasado, y de cómo las personas se ocupan y se ocupan de las facilidades y las dificultades de la vida (Ayres et al., 2016b).

La vulnerabilidad de las mujeres está fuertemente definida por un tipo de relación que las mujeres tienen con su sexualidad y con ella misma, cuya marca ha sido la subordinación al deseo masculino. Esta vulnerabilidad pone a las mujeres en situación de riesgo relacionados con enfermedades de transmisión sexual y el SIDA. Las poblaciones con mayor riesgo son precisamente los que están al margen del institucional, ya que rara vez se abordan y tienen acceso a servicios formales de salud, educación y servicios sociales de forma restringida. A modo de ejemplo, las mujeres que viven en la prostitución (Ayres et al. 2003).

Un considerable número de mujeres vive da prostitución en España, el Centro de Inteligencia contra el Crimen Organizado, realiza una estimación de aproximadamente 45.000 mujeres ejerciendo la prostitución. Sin embargo, la relación de la prostitución con ciertas conductas delictivas, unida a las características de clandestinidad y consiguiente invisibilidad, así como la estigmatización y la exclusión social en que se encuentran las personas en situación de prostitución, dificulta en gran medida la obtención de datos, sin que existan hasta el momento estadísticas oficiales al respecto (Barboza et al., 2017).

Según el Instituto Nacional de Estadística de la España en el año 2010 en base 2010, entre la economía ilegal está la prostitución y esta representa el 0,35\% del Producto Interior Bruto, lo que supone unos 3.700 millones de euros (de la Fuente, 2016). Sin embargo, de acuerdo con la institución Cáritas Española, basado en la atención prestada a mujeres entre 2011 y 2014, las españolas son las menos que ejercen prostitución en España, el 20\%, pero la crisis económica ha provocado un aumento del índex. También ha motivado que vuelvan a ejercer la prostitución en la calle mujeres extranjeras que la habían dejado hace 
años, pero que no han encontrado otra salida al quedarse $\sin$ trabajo $y \sin$ prestaciones y tener hijos a su cargo (Cáritas Española, 2016).

Aún siendo una actividad con una gran relevancia, tanto a nivel social como económico, se observa una falta de regulación, lo que conlleva a que las acciones del Estado y las instituciones que lo componen no sean suficientes para lidiar con esta realidad y tengan que entrar en acción diferentes actores del denominado Tercer Sector para hacer frente a las necesidades de las trabajadoras sexuales (Ramos e Pavia, 2016).

No se puede decir que el trabajo sexual sea la principal causa de aumento de Infecciones de Transmisión Sexual (ITS), aunque no se deben dejar de lado programas de prevención y atención a este sector altamente vulnerable, que por una parte se expone a la transmisión de ITS y VIH y, por otra, es foco de especulaciones que refuerzan los prejuicios y el señalamiento (Tirado Acero, 2014). Frente a esta problemática el objetivo de este trabajo fue relatar factores asociados a vulnerabilidad para VIH/SIDA en mujeres en dificultad social que ejercen la prostitución en Sevilla-España.

\section{Métodos}

La metodología aplicada es de técnicas cualitativas, consistentes en revisión de fuentes bibliográficas y relato de observaciones en un proyecto de Atención Social a Mujeres prostituidas, detección e Intervención Victimas de Trata en el Programa de una Organización No Gubernamental (ONG) de España con oficina en Sevilla. La observación de los contextos donde tiene lugar la situación de prostitución, tenían como objetivo poder contar con su voz como protagonistas de este fenómeno, en primera persona, a través de sus experiencias, opiniones, percepciones y perspectivas de futuro.

La muestra no probabilística fue seleccionada por conveniencia, compuesta por 15 participantes que cumplieron los criterios de inclusión: ser mujer, incluidas las transexuales, ejercer la prostitución en la calle o en apartamentos / casas destinados a la actividad y se excluyeron aquellas que rechazaron recibir ayuda del mismo, servicio de la ONG. Se recogieron testimonios de mujeres y transexuales en prostitución, de diferentes edades y orígenes geográficos. Se caracterizó por ser una muestra intencionada, una vez 


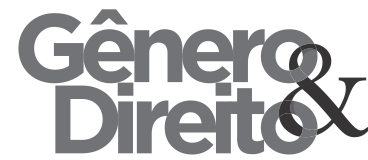

que las participantes fueron seleccionadas de acuerdo con los criterios predeterminados por el objetivo de la investigación.

La investigación se realizó en áreas urbanas y viviendas destinadas a la prostitución en Sevilla, España. Sevilla es un municipio de España, capital de la provincia homónima y de la comunidad autónoma de Andalucía, cuenta con 690 566 habitantes (Instituto Nacional de Estadística, 2016), por lo que es la ciudad más poblada de Andalucía, la cuarta de España después de Madrid, Barcelona y Valencia.

Las principales áreas de prostitución de calle son la Estrada $\mathrm{Su}$ Eminencia (localizada en el distrito Sur), Estrada Amarilla y zona Sevilla Este (ubicadas en el distrito Este-AlcosaTorreblanca). Las viviendas destinadas a la prostitución están ubicadas en todos los barrios de Sevilla. Sin embargo, en el período de recolección de datos se consiguió visitar apartamentos en el distrito de Macarena, barrio San Bernardo (ubicado en el distrito Nervión) y barrio Los Arcos (ubicado en el distrito Norte).

Una vez realizado el diseño metodológico, se procedió a la realización del trabajo de campo entre eriódico do Núcleo de Estudos e Pesquisas sobre Gênero e Direito Centro de Ciências Jurídicas - Universidade Federal da Paraíba Edição Especial - Health, Gender and Human Rights V. 7 - No 01 - Ano 2018

marzo y mayo de 2017. Se utilizó un cuaderno de campo para el registro de la información de la comunicación verbal o no verbal y observación contextual. Para desarrollar las entrevistas con mujeres en situación de prostitución, se planificó una estrategia de acercamiento.

Para la ejecución del trabajo de campo se contó con el apoyo de voluntarios de una ONG que va a calles y viviendas dedicadas a la prostitución. La ONG facilitó la aproximación de las mujeres, pues ya había un trabajo previo de intervención de los profesionales, que genera confianza o al menos de no extrañamiento entre entrevistadas y entrevistadoras. A través de los contactos previos de la ONG, fue posible detectar las principales áreas de calle y viviendas destinadas a la prostitución.

Se recogieron informaciones sobre la vida de las mujeres en prostitución, centrándose en cuestiones sociales y de salud. Las informaciones recogidas en el trabajo de campo fueron transcritas, estructuradas y analizadas a través del análisis del discurso de Minayo, (2010). Por otro lado, se presentaron resultados del trabajo de campo realizado en el escenario de esta investigación y se realizó una comparación con los resultados de otros 
estudios con población de mujeres en prostitución. Las categorías que se pretendió conocer a partir de la ejecución de las preguntas para las mujeres fueron: conocimiento de la realidad social de las mujeres involucradas en la prostitución en Sevilla; análisis de comportamientos sexuales para el riesgo de $\mathrm{VIH}$; conocimiento de las mujeres sobre las formas de prevención; identificación y diagnóstico.

Las mujeres concedieron entrevistas voluntariamente, con consentimiento informado. Se les explicó el tratamiento de anonimato y confidencialidad de la entrevista. Los investigadores pusieron a la institución la devolución de los hallazgos. Una entrevista puede ser una ocasión para revivir situaciones que muchas mujeres prefieren no hablar. Respetando el silencio en esos casos fue un paso fundamental para la confianza, por eso durante la realización de los encuentros los temas fueron abordados con total respeto, cuidando tanto las palabras y los silencios.

En este estudio se configuró no sólo un conjunto de medidas de cuidado en relación con la persona participante en la investigación, sino también una manera de estableció una relación de comunicación próxima entre la investigadora y la participante. De ahí en adelante, la investigadora expresó su interés en las experiencias de las participantes, delimitando fronteras en la relación de investigación. Es decir, la propuesta de la investigadora fue escuchar las experiencias de vida de las participantes.

\section{Resultados}

Las 15 participantes tienen nacionalidad de Ecuador (1), Brasil (2), Romania (5), España (5), Nigeria (2). El perfil mayoritario de las mujeres que ejercen la prostitución en la calle, corresponde a mujeres procedentes de Rumania (5). Mientras que, en pisos para fines de prostitución se encuentran más de España (3). Casi todas (12/15) tenían hijos(as) y tenían entre los 20 y los 40 años, con una media de edad de 30 años, la mayoría (7/10) de las mujeres inmigrantes se encontraba en situación de regularidad administrativa.

Los pisos en general son difíciles de localizar e identificar y están localizados en cualquier zona de la ciudad. Costumbre de ser pisos en alquiler, en donde pueden vivir y ejercer entre 2-5 mujeres, algunas veces 
controlados por empresarios/as. Hay mujeres que ejercen sólo durante unas horas del día y otras que viven en el mismo piso y están disponibles las $24 \mathrm{~h}$.

En cuanto a la prostitución de calle existen en Sevilla diferentes puntos en los que pueden encontrarse mujeres que ejercen la prostitución. Los principales, por número de mujeres que allí ejercen, son la Carretera de $\mathrm{Su}$ Eminencia, la Carretera Amarilla (zonas alejadas del centro urbano y ubicado en polígonos industriales) y la zona de Sevilla Este. Son zonas normalmente aisladas, con poca iluminación y ausencia de infraestructuras.

Esta aproximación de la realidad que viven las mujeres que ejercen la actividad en la calle ha sido complicada en algunas ocasiones. Se pudo ver cómo algunas de ellas mostraban renuencia a hablar y algunas incluso se rehusaron, otras dijeron que los clientes podían pensar que habríamos conectado con la policía y que los estábamos alejando. Sin embargo, se logró vivenciar un encuentro de forma fácil y tranquila con algunas de este colectivo, pues ellas ya conocían el trabajo de la ONG y pedían a los voluntarios los kits con preservativos.
Opiniones y percepciones acerca de los factores de risco para SIDA, inseguridad, el estigma, vulnerabilidad, entre otros, sentidas por la mayoría de las mujeres, se aprecian a continuación (diarios de campo, marzomayo, 2017):

Todas las mujeres abordadas en el contexto de proyecto, afirmaran que estaban en prostitución por no poder optar a otro medio para conseguir dinero. Algunas (12) para sobrevivir o para sustentar sus familias, otras (3) por el consumo de sustancias, e indican que hubieran preferido poder optar por otro "empleo". Algunas de las entrevistadas coinciden en afirmar que la crisis económica ha disminuido la demanda e incrementado la competencia entre las mujeres. En este sentido, la prostitución se rige en los mismos términos en los que funcionaría el mercado de consumo.

Se ha podido observar que, el tema de las drogas es algo que sólo tres se atrevieron a hablar en primera persona, reconociendo su uso $\mathrm{y}$ puntualmente, su abuso. Lo más normal es que se hable de los problemas con el abuso de drogas de las otras mujeres con las que se comparte espacio. Algunas mujeres entrevistadas también 


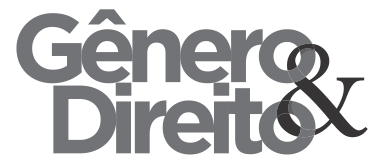

Periódico do Núcleo de Estudos e Pesquisas sobre Gênero e Direito

Centro de Ciências Jurídicas - Universidade Federal da Paraíba Edição Especial - Health, Gender and Human Rights

V. 7 - No 01 - Ano 2018

ISSN | 2179-7137 | http://periodicos.ufpb.br/ojs2/index.php/ged/index

relacionan el contexto de calle con el inicio en el consumo.

Yo igual consigo otro trabajo y salgo de esto, de limpiar casas, camarera, lo que sea, un día saldré de esto, tengo un currículo, ninguna le gusta esta profesión. (E01)

Algunas chicas jóvenes bajan muchas veces el precio porque las cosas están difíciles; y ya tiene algunas que aceptan ir sin preservativo, yo miro eso en los anuncios. (E11)

Yo no comprendo jóvenes que no tienen necesidad de dinero pero entran en la prostitución para comprar un móvil caro o ropas de grife, yo tengo lo que comer, pero estoy trabajando para pagar mi alquiler y sustentar mis hijos. (E08)

Las de la calle están más rápido en la droga. (E06)

Fue observado un estigma entre ellas, en la propia organización del territorio en el que ellas ejercen, además esconden esta actividad a sus familias. Las mujeres atendidas por la ONG, proceden de entornos de pobreza estructural, lugares donde faltan los recursos básicos, de lugares en conflicto o donde han sufrido violencia. Las africanas tenían más miedo de ser denunciadas por no tener su situación administrativa resuelta. Las barreras de idioma y cultural también son mayores entre las africanas.

En otra zona tiene las que son más baratas y utilizan droga, en otra las travestis y tiene una calle con más nigerianas. (E07)

Yo no puedo compartir piso con una persona que no quiere que llegue tarde o haga barullo o traga hombres para mi habitación, yo te dice que trabajo cuidando de un anciano pela noche y llego en casa cuando la hija llega de trabajo para quedar con ello. (E09)

No quiero informar mi nombre es que mi familia, mi madre, mis hijos no saben, no pueden saber. (E01)

No es fácil, yo tomé puntos en el cuello porque lo tío me llamó a un lugar secreto y me cortó con un cuchillo, pero estoy aquí de nuevo, tengo que trabajar, así es la vida. (E10)

Sobre las condiciones maternas fue observado relato que más que considerar su actividad un riesgo para su embarazo, su embarazo pone en riesgo su actividad por lo que los abortos se 
vuelven rutinarios. Doce de las trabajadoras sexuales entrevistadas son madres de uno o varios hijos/as y no tienen mucho tiempo para los cuidados maternos, una incluso reclama la falta de ayuda del marido con la creación de los hijos.

Yá es mi tercero aborto, no se preocupe, yo no necesito de ayuda, creo que estoy con 3 o 4 meses, voy retirar porque tengo que trabajar, no puedo trabajar embarazada (E8)

Mi marido está desempleado, no trabaja, pero soy yo la responsable por limpiar mi casa y mis hijos y perdí la ayuda social porque la trabajadora social miro mis hijos sucios y la casa sucia también. ¿Pero como voy limpiar se yo trabajo más de doce horas por día y llego demasiada cansada en mi casa? (E1)

Algunas mujeres contestaran nunca haber realizado un teste diagnóstico de VIH y hubieran relatos diversos cuanto al motivo para ninguna realización, entre ellos: creer que no corría riesgo de infectarse, miedo de discriminación en cuanto al resultado del test, creer en la fidelidad de su cliente fijo o compañero íntimo, dificultad de acceso a la prueba por la poca disponibilidad de locales fuera del ámbito hospitalario y falta de unidades de salud que atiendan en horarios alternativos.

Yo no he ido al médico desde que llegué, si me pasa algo voy a la farmacia, me compro algo. (E02)

No tengo tarjeta sanitaria porque no puedo mostrar mis documentos, estoy aquí ilegal. Ustedes no hacen la prueba de VIH? Donde vivía tenía una institución que hacía en la calle (E12)

Tiene una en esta zona que todas sabemos que tiene AIDS y está haciendo sexo sin preservativo, eso me asusta. (E04)

Yo utilizo preservativo siempre pero tiene veces que se rota por cuenta de calentador o acondicionado y me quedo muy preocupada pero no hice la prueba para VIH. (E11)

Yo no necesito de consulta ginecológica, no tengo nada, no tengo dolor o síntoma ningún, se tengo VIH prefiero no saber. (E07)

En cuanto al uso de condones, cuando mantienen relaciones con clientes tienden realizar sexo con 
protección, mientras que con su pareja estable no es una práctica habitual. El no uso del preservativo también suele extenderse a clientes habituales con los que aunque no sean la pareja estable sí se da un vínculo emocional o de cierta cercanía.

Con mi pareja no lo uso porque sé que no tiene nada, pero con los clientes siempre utilizo. (E05)

Yo tengo mis clientes habituales que confí y tiene horario fijo conmigo, eses as veces no utilizo preservativo y creo que es responsable porque tiene hijos y mujer, sé que él no tiene nada. (E08)

Yo solo llevo para mi habitación mi cliente fijo porque es mayor no es joven y confío, pero los otros tienen que pagar hoteles. (E03)

\section{Discusión}

Lo perfil de las mujeres del presente estudio es semejante a el informe de la institución Cáritas Española, en 2016, cuando apunta cuanto a la nacionalidad de las mujeres que ejercen la prostitución en España. Nigeria, Brasil, Rumanía, República Dominicana y Colombia estaban entre los países de origen más frecuentes. Según los autores Alles e Cogo, 2016, la temática de la migración femenina, especialmente cuando se refiere a las mujeres que migran de países de países subdesarrollados o en desarrollo a países de Europa Occidental y Estados Unidos, aparece frecuentemente relacionada con las actividades en el ámbito del mercado transnacional del sexo. Sin embargo, es necesario distinguir conceptualmente tráfico de personas de prostitución para evitar la profundización de la estigmatización y marginación de los sujetos implicados en el trabajo sexual.

Entender el contexto en el cual esas mujeres crecieron es fundamental y se desarrollaron para comprender la razón por estar en situación de prostitución. Por lo tanto, se entiende la importancia de diferenciar la prostitución voluntaria de la forzada, en el sentido de respetar la libre elección de las que deciden ejercer la actividad y luchar contra la prostitución forzada, pues constituye una violación de los derechos fundamentales de esas mujeres. En caso de las que han elegido, pero soliciten salir de dicha situación a través de intervención, se deben ayudarlas. Si no desean salir, se debe favorecer el 
mejor y más seguro desarrollo de esa actividad (Pavía et al., 2017).

La crítica a la dicotomía de la prostitución forzada contra la prostitución voluntaria está basada en el refuerzo del estigma. Dividiendo a aquellas mujeres que fueron "forzadas" a entrar en el mercado del sexo o por terceros o por condiciones de pobreza extrema, de aquellas que entran en el mercado por libre elección (Ribeiro, 2016). Las trabajadoras del sexo viven un estigma social diario para romper con los mandatos atribuidos a las mujeres en relación a la sexualidad. Explicar un servicio sexual a cambio de dinero, implica sanción social, que van desde comentarios despectivos vecinos $\mathrm{y}$ familiares hasta el abuso de cliente y extorsión de policías (Sanchés, 2016).

Las migrantes trabajadoras del sexo son objeto del triple estigma de criminal, puta e inmigrante, volviéndose blanco de un trato inhumano $\mathrm{e}$ irrespetuoso. En el discurso sobre los migrantes, es ampliamente asumido que las mujeres pobres de otros países entran en el trabajo sexual como una forma de supervivencia. Wesenbeeck (1994), por ejemplo, reconoce que una grave necesidad económica en una situación caracterizada por pocas oportunidades sociales parece ser la principal motivación para las mujeres en el mundo no occidental entrar en la prostitución. No obstante, la prostitución profundiza aún más esta situación social, porque las estigmatiza, aísla, margina y les limita el acceso a los recursos. Las mujeres latinoamericanas aún poseían la ventaja de manejo con el español a diferencia de las mujeres de origen africano.

Todas estas cuestiones citadas, también son latentes en el marco normativo de la prostitución en España. En ese sentido, se nota que en España, la prostitución es una actividad que está en una situación de ilegalidad. Aunque no existe en el sistema jurídico del país ningún reglamento que plantee cuestiones relacionadas con el desarrollo de esta actividad como una profesión o trabajo, se pueden encontrar otras cuestiones planteadas en ciertas áreas del sistema jurídico. Las políticas públicas y normas encontradas están dirigidas, generalmente, a la búsqueda de la erradicación de la prostitución, evaluando a las mujeres en la prostitución como víctimas, y que por otro lado, se debe obtener la reinserción en la sociedad (Pavía et al., 2017).

En la ciudad de Sevilla, se sigue la Portaria Ordenanza del 2011, para 
luchar contra la prostitución y la trata con fines de explotación sexual. Desde el primer momento, establece como objetivo principal la lucha contra la prostitución y el tráfico para fines de explotación sexual en la ciudad de Sevilla. Todo con la intención de preservar a lugares públicos y, para ello, se establecieron diferentes mecanismos en esos espacios que ayudan a prevenir su explotación, también reforzó el control de los lugares donde hay la realización de la prostitución. Esta ordenanza local que multa al cliente de prostitución callejera ha sido implementada por causa de las molestias por la prostitución en la calle. Sólo responde a la cara más visible de la prostitución y con el único objetivo de ocultarla.

\section{La prostitución callejera} entraña riesgos para la amenaza de la gran pandemia del VIH, con el peligro de que se pueda producir por la transmisión del virus a través del contagio sexual o por el intercambio de jeringuillas pues algunas son drogodependientes. Violencias sufridas por personas sometidas a condiciones de trata pueden ocasionar consecuencias físicas, psicológicas, sociales y de salud sexual y reproductiva graves, como por ejemplo; contraer infecciones de transmisión sexual, VIH/SIDA.

Dentro de la jerarquía de la cultura sexual prevalente, el intercambio sexual por dinero es una práctica castigada por el "estigma de puta" de las mujeres que representa para trabajadoras mujeres del sexo se exponer a condiciones de exclusión, discriminación y violencia. Estas sanciones son aplicadas por la familia y la comunidad y el Estado, a través de la prohibición, omisión o falta de protección para las personas que se involucran en el trabajo sexual (Sánchez, 2016).

La experiencia de la violencia puede aumentar el comportamiento de riesgo para el VIH. En nivel emocional y psicológico la mayoría de las mujeres sufren situaciones muy extremas, en donde el miedo, y as veces el pánico, se convierten en algo cotidiano, algo que aprenden a llevar, a normalizar, con costes elevados en términos de equilibrio físico, psíquico y emocional (Lima et al., 2016).

Las mujeres en situación de prostitución y dificultad social tienen que lidiar ante estas situaciones de violencia y exclusión citadas. Asimismo tienen que sobrellevar condiciones 
maternas, ya que son varias las trabajadoras sexuales que son madres de uno o varios hijos/as e incluso o en algunos casos se encuentran cursando su primer embarazo, lo cual hace que este proceso se convierta en muchas de ellas en experiencias negativas que provocan incertidumbre de su futuro (Zanabria et al., 2016). Adicionalmente, según la división de trabajo sexual de lo sistema patriarcal en lo mundo capitalista, apenas la mujer fornece en su matrimonio el trabajo gratuito de creación de los hijos y en lo cotidiano de las actividades domésticas (Biroli, 2016).

Para las mujeres, las normas de género asocian a práctica de sexo al amor y à reproducción, criando el supuesto de la maternidad como deseo de todas. Las mujeres que rompen estas reglas, ejerciendo la sexualidad como un trabajo remunerado, que rechazan un embarazo, causan un aborto involuntario, o que tienen VIH, a menudo sin haber violado ninguna regla, se ven particularmente afectados por la estigmatización y sus consecuencias para la salud (Villela e Monteiro, 2015). Además, la prostitución callejera representa peores condiciones y lleva a múltiples riesgos que pueden afectar su salud y del producto de la gestación.
60

Sin embargo, fue observado un estigma entre ellas, en la propia organización del territorio en el que ellas ejercen, además esconden esta actividad a sus familias. La falta de reconocimiento de la trabajadora sexual tiende a producir un sufrimiento patógeno. Toma nota de la falta de reconocimiento social por los padres, la familia, la sociedad incluso el propio gobierno eso no le da sus derechos laborales (Rodrigues Filho, 2014).

La posibilidad de que los derechos se hagan realidad dependerá del tipo de organización social y política, si éste permite o impide a las personas tener derecho a bienes públicos. El autor creer que pocos ámbitos como el de la salud son tan propicios para entender que el acceso efectivo a ciertos recursos es fundamental para el ejercicio de la autonomía (Egan e Fernández, 2016). Tener derecho a bienes públicos es un requisito de la dignidad plena.

Aunque, la población residente en Andalucía tenga derecho a la asistencia sanitaria gratuita a cargo del sistema público andaluz, el horario de funcionamiento de las unidades, la rutina de la vida y el temor de ser atendidas, debido al estigma, alejan las prostitutas de los servicios de salud. No obstante, las 
deficiencias en el acceso a los servicios de salud para las mujeres de este segmento han obstaculizado cada vez más datos reales, que nos permiten conocer cuál es la situación de las mujeres en la prostitución sobre el diagnóstico de las infecciones de transmisión sexual.

En este sentido, es necesario conocer cuáles son las barreras e incentivo a las pruebas y la periodicidad. El programa conjunto de las Naciones Unidas para el VIH/SIDA enfatizó que la prueba y asesoramiento para VIH es la puerta de entrada para lo tratamiento y funcionaria con un salvavidas para las mujeres en situación de prostitución, así mismo, solo mitad de esas personas en todo el mundo conocía su estado serológico. La Organizacion Mundial de la Salud refuerza la énfasis en la importancia de la pruebas y el asesoramiento para lo VIH. Por esta razón recomienda que la prueba y el asesoramiento debe hacerse en forma rutinaria ofertados para las poblaciones llaves, en las comunidades y en todos los estabelecimientos clínicos (World Health Organization, 2014).

Lo que acontece es que, el acceso a la información referida a la prevención y tratamiento de ITS y VIH en el trabajo sexual con o sin consentimiento, tiene limitaciones, pues no es homogéneo para toda la población, por restricciones presentes que se asocian a la estigmatización de la cual es objeto el trabajo sexual (Tirado Acero, 2014). El estigma también dificulta la prevención del VIH/Sida, retrasa la búsqueda por el diagnóstico y fomenta as consecuencias negativas de un diagnóstico tardío. Más aún, interfiere en la calidad de vida pos-diagnóstico, haya vista las dificultades da condición serológica y el aislamiento social.

En cuanto al uso de condón se sabe que es importante en toda relación sexual, por causa del riesgo de contraer alguna ITS como el VIH. Es importante recalcar el uso del preservativo en las trabajadoras sexuales, porque es una población que se encuentra en alto riesgo por la actividad diaria que realizan. Por otra parte el uso del condón es aceptado totalmente con los usuarios o clientes, no obstante si hablamos de sus relaciones sexuales con sus parejas estables (novios, maridos) es prácticamente nulo y esto se debe al factor confianza que existe de parte de ellas.

Sin embargo, las trabajadoras sexuales se exponen en total riesgo en el momento de mantener relaciones con sus 
parejas regulares que podrían tener otras parejas y ellos llegarían a ser vehículos de ITS. Las relaciones no-comerciales de las mujeres en prostitución, muchas veces son olvidadas. El estudio de Argento, et al. (2014), mostró uso inconsistente de preservativo para sexo vaginal y /o anal con compañero íntimo por mujeres en prostitución y una alta prevalencia de violencia también.

Además del conjunto de la vulnerabilidad individual en cuanto a los comportamientos sexuales, los factores sociales actúan como fuertes predictores del riesgo de contraer IST (Bajaj et al., 2017). Lo empoderamiento de la comunidad puede ser un poderoso determinante para protección contra lo risco de VIH entre los profesionales de sexo. Estudios realizado en Brasil y Vancouver indicaran que factores socioculturales externos a lo ambiente de trabajo. Autonomía e inserción en redes sociales, también tienen asociaciones significativas con el uso del preservativo (Argento et al., 2016; Lippman, 2012).

Argento et al., en Vancouver, modelaron el impacto de la cohesión social en la recusa del cliente en utilizar preservativo entre profesionales del sexo. En Brasil, el estudio de Lippman resultó evidente que la cohesión social elevada estaba inversamente asociada al número de actos sexuales sín protección en la semana anterior $(\operatorname{IRR}=0,80 ; \mathrm{p}<$ $0,01)$ y la participación de mujeres en redes sociales estaba asociada a una reducción en la frecuencia de sexo sín protección.

El reconocimiento de la relación entre la vulnerabilidad, procesos de estigmatización y los ejes de desigualdad social y de género sugiere un mayor acceso a la salud e implica intervenciones estructurales y culturales, que sean capaces de promover la autonomía de la mujer y garantizar sus derechos sexuales y reproductivos. Identificar factores de vulnerabilidad es fundamental para las intervenciones que garantan derechos humanos y controle del VIH.

\section{Conclusiones}

Los componentes de la vulnerabilidad social se han identificado en las historias de la vida de las mujeres mediante el análisis de las historias de vida contadas por ellas. Las variables más identificadas fueron malas condiciones de vida, la condición femenina relacionada con las relaciones de género y la situación económica. Se 
verificó la vulnerabilidad para abuso de drogas e violencia, configurándose una mayor vulnerabilidad al HIV/SIDA.

La condición femenina marcada por la mala situación económica se consideró, en este estudio, como un determinante de la vulnerabilidad social evidenciado por factores asociados, como el uso de drogas, sometimiento a la pareja sexual, la dependencia financiera y económica y la cuestión de la supervivencia. Aún consciente de los riesgos y la necesidad de sexo seguro, algunas mujeres en el estudio se someten a la voluntad de la pareja sexual, poniendo de relieve la falta de poder de negociación sobre el uso de preservativos durante las relaciones sexuales, lo que conduce a no protección del cuerpo y la vulnerabilidad de la mujer a las infecciones de transmisión sexual.

La vulnerabilidad programática fue identificado en la falta de conocimiento de los programas de prevención de ITS / SIDA, en los aspectos de la maternidad y la salud reproductiva y el impacto del diagnóstico del VIH. La falta de conciencia de algunas mujeres en las prácticas de prevención de infecciones de transmisión sexual es un factor muy importante que puede causar la vulnerabilidad a un diagnóstico de VIH / SIDA entre estas mujeres. Independientemente de los posicionamientos acerca de la regulación o abolición de la prostitución, es importante que las prostitutas sean reconocidas como trabajadoras $y$ ciudadanas y tengan sus derechos asegurados

\section{Referências Bibliográfica}

Alles, Natália Ledur; Cogo, Denise (2017), "Mídia e Migração Feminina (In) distinções entre trabalho sexual e tráfico de pessoas", Périplos: Revista de Pesquisa sobre Migrações, 2(2), 1-17. Consultado a 05.07.2017, em http://periodicos.unb.br/index.php/obmi gra_periplos/article/view/25526/18254

Argento, Elena et al. (2014), "High prevalence and partner correlates of physical and sexual violence by intimate partners among street and off-street sex workers", PLoS One, 10(7), e102129. Consultado a 08.06.2017, em http://dx.doi.org/10.1371/journal.pone. 0102129. 
Argento, Elena et al. (2016), "Social

Cohesion Among Sex Workers and

Client Condom Refusal in a Canadian

Setting: Implications for Structural and Community-Led Interventions", AIDS Behav, 20(6), 1275. Consultado a 01.06.2017,

em

http://dx.doi.org/10.1007/s10461-015$1230-8$

Ayres, José Ricardo de Carvalho Mesquita et al. (2003) O conceito de vulnerabilidade e as práticas de saúde: novas perspectivas e desafios. In: Czeresina, Dina; Freitas, Carlos Machado, Promoção da saúde: conceitos, desafios, tendências, 1 ed, Rio de Janeiro: Fiocruz, 117-138.

Ayres, José Ricardo de Carvalho Mesquita et al. (2016a), "O conceito de Vulnerabilidade e as Práticas de Saúde: novas perspectivas e desafios", in: Czeresina, Dina; Freitas, Carlos Machado, Promoção da Saúde: conceitos, reflexões, tendências, Rio de Janeiro: Fiocruz, 121-43.

Ayres, José Ricardo de Carvalho Mesquita et al. (2016b), Risco, vulnerabilidade e práticas de prevenção e promoção da saúde. In: Campos,
Gastão Wagner de Sousa et al., Tratado de Saúde Coletiva, 2.ed, São Paulo, 375418.

Barboza, Lourdes, et al. (2014), "La Trata de Mujeres y Niñas con fines de explotación sexual en el Paraguay 20102013”, Asunción: Organización Internacional para las Migraciones. Consultado a 13.05.2017, em http://hdl.handle.net/20.500.11788/1472

Biroli, Flávia (2016), "Divisão sexual do trabalho e democracia", Dados-Revista de Ciências Sociais, 59(3), 719754. Consultado a 13.05.2017, em https://dx.doi.org/10.1590/00115258201 690

Cáritas Española (2016), "Reflexiones y marcos de acción: La prostitución desde la experiencia y la mirada de Cáritas", Madrid: Cáritas Española Editores Consultado a 25.05.2017, em http://www.caritasvitoria.org/datos/docu mentos/CARITASProstitucion2016.pdf

de la Fuente, Angel (2016), "Series enlazadas de Contabilidad Regional para España, 1980-2014”, Madrid: FEDEA, Estudios sobre la Economía Española. Consultado a 25.05.2017, em 
http://documentos.fedea.net/pubs/eee/ee e2016-35.pdf

Egan, Luis Alberto Villanueva; Fernández, Miguel Ángel Lezana (2016), "Derechos Humanos y Derecho a la Salud. Los desafíos contemporáneos", Revista CONAMED 21(4). Consultado a 10.04.2017, em http://www.dgdi-

conamed.salud.gob.mx/ojs-

conamed/index.php/revconamed/article/ view/513

Instituto Nacional de Estadística (2016). “Cifras oficiales de población resultantes de la revisión del Padrón municipal a 1 de enero". Página consultada a 26.05.2017, em http://www.ine.es/dynt3/inebase/es/inde $\mathrm{x} . \mathrm{html}$ ? padre $=517 \& \mathrm{dh}=1$

Lima, Francisca Sueli da Silva et al. (2017), "Factors associated with violence against female sex workers in ten Brazilian cities", Cad Saúde Pública, 33(2), e00157815. Consultado a 20.04.2017, em http://dx.doi.org/10.1590/0102-

$311 \times 00157815$.

Lippman, Sheri et al. (2012), "Findings from Encontros: a multi-level STI/HIV intervention to increase condom use, reduce STI, and change the social environment among sex workers in Brazil", Sex Transm Dis, 39(3), 209213. Consultado a 20.04.2017, em http://dx.doi.org/10.1097/OLQ.0b013e3 $1823 b 1937$.

Mann, Jonathan et al. (1993), “A aids no mundo", in: História Social da AIDS, ABIA; IMS; UERJ.

Minayo, Maria Cecília de Souza (2010), "Pesquisa social: teoria, método e criatividade". Petrópolis: Vozes.

Ministerio de Sanidad (2016). "Servicios Sociales e Igualdad. Servicios Sociales e Igualdad 2015-2018; plan integral de lucha contra la trata de mujeres y niñas con fines de explotación sexual, 20152018”, España: Ministerio de Sanidad. Página consultada a 20.04.2017, em http://www.violenciagenero.msssi.gob.e s/planActuacion/planContraExplotacion Sexual/

Ordenanza para luchar contra la prostitución y la trata con fines de explotación sexual en la ciudad de Sevilla, 2011. Boletín Oficial del Estado de la provincia de Sevilla. 
Periódico do Núcleo de Estudos e Pesquisas sobre Gênero e Direito Centro de Ciências Jurídicas - Universidade Federal da Paraíba Edição Especial - Health, Gender and Human Rights V. 7 - No 01 - Ano 2018

ISSN | 2179-7137 | http://periodicos.ufpb.br/ojs2/index.php/ged/index

Pavía, Rafael Barroso (2017). Tirado Acero, Misael (2014), "El trabajo "Intervención Social Con Mujeres Que Ejercen La Prostitución", in: Libro de Intervención Social con Colectivos Vulnerables. Editorial Dykinson.

Ramos, Nuria Cordero; Pavía, Rafael Barroso (2016), “¿Quién Protege los Derechos de las Trabajadoras Sexuales? Revisión y Análisis de la Legislación Española en Materia de Trabajo Sexual", Themis: Revista da Esmec, 14, 249-272. Consultado a 30.05.2017, em http://revistathemis.tjce.jus.br/index.php /THEMIS/article/view/537

Rodrigues Filho, Luciano Ferreira (2014), "Prostituição: um estudo sobre as dimensões de sofrimento psíquico entre as profissionais e seu trabalho", Revista Científica da UEM: Série Ciências da Educação, 1(1), 114-123. Consultado a 30.05.2017, em http://www.revistacientifica.uem.mz/ind ex.php/rcce/article/view/55

Sánchez, Bertha (2016, "Menos prejuicios morales y más derechos laborales", Cuaderno Jurídico y Político, 1(4), 116-124. sexual desde una perspectiva de los derechos humanos: implicaciones del VIH/sida e infecciones de transmisión sexual", Civilizar Ciencias Sociales y Humanas, 14(27), 97-110. Consultado a 27.05.2017, em http://www.scielo.org.co/pdf/ccso/v14n 27/v14n27a07.pdf

Vanwesenbeeck, Ine (1994). Prostitutes' well-being and risk. Amsterdam: VU University Press.

Villela, Wilza Vieira; Monteiro, Simone (2015), “Gênero, estigma e saúde: reflexões a partir da prostituição, do aborto e do HIV/aids entre mulheres". Epidemiologia e Serviços de Saúde, 24(3), 531-540. Consultado a 26.05.2017, em http://www.scielo.br/pdf/ress/v24n3/223 7-9622-ress-24-03-00531.pdf

World Health Organization (2014), “The Gap Report. Geneva, Switzerland: United Nations on HIV/AIDS”. Página consultada a 26.05.2017, em http://www.unaids.org/en/media/unaids/ contentassets/documents/unaidspublicat ion/2014/UNAIDS_Gap_report_en.pdf. 
World Health Organization (2016),

"Sexually transmitted infections".

Página consultada a 25.05.2017, em http://www.who.int/mediacentre/factshe ets/fs110/en/.

Zanabria Cosar, M. K. (2016). Actitudes y experiencias relacionadas a la maternidad en las trabajadoras sexuales atendidas en el Centro de Salud Madre Teresa de calcuta. Lima, 2015. Lima: Universidad Nacional Mayor de San Marcos. Consultado a 13.04.2017, em http://cybertesis.unmsm.edu.pe/handle/c ybertesis/4730 